\title{
Experimental validation in optical fibers of multiple Fermi-Pasta-Ulam-Tsingou recurrences theory
}

\author{
Corentin Naveau ${ }^{1}$, Pascal Szriftgiser ${ }^{1}$, Alexandre Kudlinski ${ }^{1}$, Matteo Conforti ${ }^{1}$, Stefano Trillo $^{2}$, \\ and Arnaud Mussot ${ }^{1}$ \\ 1. Univ. Lille, CNRS, UMR 8523-PhLAM - Physique des Lasers Atomes et Molécules, F-59000 Lille, France \\ 2. Department of Engineering, University of Ferrara, Via Saragat 1, 44122 Ferrara, Italy
}

Fermi-Pasta-Ulam-Tsingou (FPUT) recurrence process describes the ability of a multimodal system experiencing complex nonlinear dynamics to come back to its initial state, instead of reaching thermalization. In optics, it has particularly been investigated in the context of modulation instability (MI) [1], which plays a key role in many nonlinear phenomena such as rogue wave formation or supercontinuum generation. Recently, we reported a novel experimental setup [2] consisting in a heterodyne time-domain detection system which allows to perform along an optical fiber distributed measurement in phase and intensity of a pump wave and its modulation sidebands. Thanks to an active compensation of the losses, it enabled the observation of two FPUT recurrences cycle for the first time (Fig. 1(b,c)). Moreover, Grinevich and Santini recently reported [3] an advanced theoretical model to predict the characteristics of these recurrences which have been verified experimentally in a 3D photorefractive crystal [4]. Based on the setup described in [2], we report here its verification in an optical fiber.
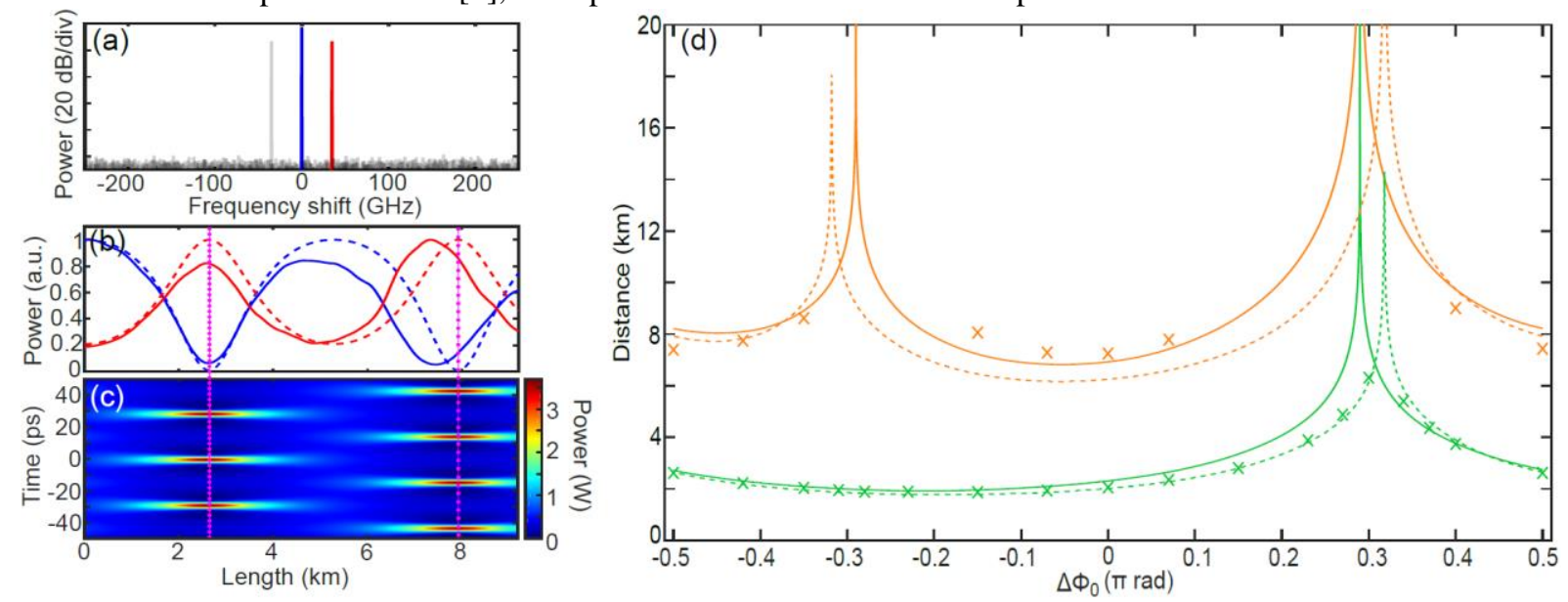

Fig. 1 (a) Simulated input spectrum. (b) Longitudinal evolution for $\Delta \Phi_{0}=-\pi / 2$ of the pump power (blue curves) and the signal one (red curves). Solid lines correspond to exprimental recordings and dashed ones to simulations. Plots are normalized to their respective maxima. (c) Corresponding evolution in the time domain from NLSE. The magenta dashed lines highlight the postion of the maximum compression points. (d) Position of the first (green) and second (orange) pump minima. Crosses correspond to experiments, dashed lines to the NLSE and solid ones to the theoretical model. Parameters : fibre length $L=9.2 \mathrm{~km}$, group velocity dispersion $\beta_{2}=-19 \times 10^{-27} \mathrm{~s}^{2} / \mathrm{m}$, nonlinear coefficient $\gamma=1.3 \times 10^{-3} / \mathrm{W} / \mathrm{m}$, pump power $P_{p}=470 \mathrm{~mW}$, signal to pump ratio $-10.4 \mathrm{~dB}$, signal detuning $35 \mathrm{GHz}$ from the pump.

The theoretical model of Grinevich and Santini predict that changing the initial relative phase $\Delta \Phi_{0}$ between the signal and the pump wave (see the input spectrum in Fig. 1(a)) strongly affects the positions of the maximum compression points which is really close to the positions of the pump minima (see the magenta dashed lines in Fig. $1(\mathrm{~b}, \mathrm{c})$ ). Varying $\Delta \Phi_{0}$ from $-\pi / 2$ to $\pi / 2$, we checked this experimentally in a $9.2 \mathrm{~km} \mathrm{SMF-28} \mathrm{fibre.} \mathrm{The} \mathrm{positions}$ of the pump minima are displayed in Fig. 1(d), with green crosses for the first minimum and orange ones for the second, the theoretical predictions of Grinevich and Santini superimposed in solid lines and numerical simulations in dashed lines. We obtain a good overall agreement, especially for the first minimum. Discrepancies appearing at the second one might be explained from the facts that (i) theoretical calculations assume a weak perturbation while we used a relatively strong modulation (here of $-10.4 \mathrm{~dB}$ of signal to pump ratio) and (ii) loss compensation is not perfect. In summary, we verified for the first time the validity of theoretical predictions of Grinevich and Santini about FPUT with two recurrences in an optical fiber. Still in the framework of the theoretical model, we also investigated how an initial asymmetry of the amplitude of the modulation sidebands affect the recurrences.

\section{References}

[1] G. Van Simaeys, Ph. Emplit, M. Halterman, Experimental Demonstration of the Fermi-Pasta-Ulam Recurrence in a Modulationally Unstable Optical Wave, Phys. Rev. Lett. 87, 033902 (2001).

[2] A. Mussot, C. Naveau, M. Conforti, A. Kudlinski, F. Copie, P. Szriftgiser, and S. Trillo, Fibre multi-wave mixing combs reveal the broken symmetry of Fermi-Pasta-Ulam recurrence, Nat. Phot. 12, 303-308 (2018).

[3] P. G. Grinevich and P. M. Santini, The exact rogue wave recurrence in the NLS periodic setting via matched asymptotic expansions, for 1 and 2 unstable modes, Phys. Lett. A 382, 973 (2018).

[4] D. Pierangeli, M. Flammini, L. Zhang, G. Marcucci, A. J. Agranat, P. G. Grinevich, P. M. Santini, C. Conti and E. DelRe, Observation of Fermi-PastaUlam-Tsingou recurrence and its exact dynamics, Phys. Rev. X 8, 041017 (2018). 